\title{
Study on the Method of Identifying Ultrafine Bubbles Using Ultrasound under the Presence of Solid Nanoparticles
}

\author{
Naoya YAMAWAKI ${ }^{1}$, Hayato OKUMURA ${ }^{2}$, Shigenori AKAMATSU ${ }^{2}$, Yusuke NishIUCHI ${ }^{2}$, and Takashi HATA ${ }^{2 *}$ \\ ${ }^{1}$ Advanced Course of Material Engineering, National Institute of Technology (KOSEN), Kochi College, 200-1 Monobe, Nankoku, \\ Kochi, 783-8508, Japan \\ ${ }^{2}$ Department of Social Design Engineering, National Institute of Technology (KOSEN), Kochi College, 200-1 Monobe, Nankoku, \\ Kochi, 783-8508, Japan
}

\begin{abstract}
The method of making $\mathrm{OH}$ radicals generated by the collapse of ultrafine bubbles as an indicator when applying ultrasound was examined as the method of identifying ultrafine bubbles less than $1 \mu \mathrm{m}$ in diameter under the presence of mixed solid nanoparticles. First, potassium iodide $(\mathrm{KI})$ method was used to evaluate $\mathrm{OH}$ radicals. Since difference in absorbance due to $\mathrm{OH}$ radicals generated by the collapse of ultrafine bubbles is very small when using air as contained gas, evaluation using the $\mathrm{KI}$ method was difficult; however, a distinct difference in absorbance was confirmed by increasing the amount of $\mathrm{OH}$ radicals generated by using ozone as contained gas. Moreover, the higher the ultrasound frequency, the greater the difference in absorbance. On the other hand, a luminescence phenomenon related to the collapse of ultrafine bubbles by ultrasound was confirmed with sonoluminescence observation, confirming further that the luminescence intensity of water added with solid particles of the same diameter and number density as those of ultrafine bubbles is smaller than that of ultrafine bubble water.
\end{abstract}

\section{Introduction}

The International Standardization (ISO) has defined to call air bubbles with less than $100 \mu \mathrm{m}$ diameter, which were hitherto called micro-nano bubbles, as "fine bubbles" whose breakdown: "microbubbles" with 1 to $100 \mu \mathrm{m}$ diameter and "ultrafine bubble" with less than 1 $\mu \mathrm{m}$ diameter. Because these fine bubbles reveal physical and chemical properties significantly different from the $100 \mu \mathrm{m}(=0.1 \mathrm{~mm})$ or more sized bubbles that can be familiarly found in everyday life, they can be applied not only to engineering fields such as gas-liquid reaction, but also to a wide range of industrial fields such as cleaning and sterilization, waste water processing, bioprocess, pharmaceuticals/preparations, food, and agriculture and fishery industries, making it increasingly important not only, of course, to analyze the academic basic phenomena, but also to implement them in the society (Terasaka, 2014).

In particular, while the presence of ultrafine bubbles (bulk-nano bubbles) defined as less than $1 \mu \mathrm{m}$ among fine bubbles is confirmed by a scanning electron microscope (Ohgaki et al., 2010) or a transmission electron microscope (Uchida et al., 2011), the laser diffraction/scattering method and the nanoparticle tracking analysis method (Kobayashi et al., 2014), which are currently used for measuring particle distribution and number density, are employed to evaluate particles of 1 $\mu \mathrm{m}$ or less including solid particles. In practice, using ultrapure or pure water in a clean room, and further introducing air through a hollow fiber filter or pure gas from a cylinder for the contained gas to avoid mixing of impurities as far as possible; however, since most actual industries do not use only ultrapure or pure water, but use water containing impurities to a certain extent, it becomes an urgent requirement in industrial and academic items connected to it to evaluate ultrafine bubbles under the conditions with impurities. In order to meet this requirement, a resonance mass measurement method capable of distinguishing between particles with positive and negative buoyancy using the specific frequency of substance was proposed. Although this method judges as ultrafine bubbles by regarding that particles with positive buoyancy are lighter than water; in fact, however, since the weight of impurities is not uniform, it is not possible to regard all the particles with positive buoyancy as ultrafine bubbles. On the other hand, by focusing on the sample, evaluation to estimate ultrafine bubbles from the difference in the measurement results before and after removing ultrafine bubbles using freeze-thaw, ultracentrifugation method, or the like is examined; however, this time, on the contrary, there is a problem that all the ultrafine bubbles cannot be removed. In other words, it becomes necessary and important to develop an analysis technology capable of identifying only ultrafine bubbles even in the presence of impurities. For this reason, a method of applying ultrasound to ultrafine bubble water to evaluate the presence of

* Corresponding author: thata@ms.kochi-ct.ac.jp 
ultrafine bubbles from the velocity and attenuation of ultrasound has been proposed in recent years (Leroy and Norisue, 2016).

Although the method of applying ultrasound is the same as the previous proposal as a method capable of qualitatively identifying the presence of ultrafine bubbles even under the presence of impurities, this study focused on free radicals generated by the collapse of ultrafine bubbles when applying ultrasound. The previous research has confirmed that ultrafine bubbles exist stably, but collapse by external stimuli and generate $\mathrm{OH}$ radicals (Tada et al., 2014). Moreover, there is also a simulation result that $\mathrm{OH}$ radicals are not generated only by the shrink of ultrafine bubbles (Yasui et al., 2016). In this study, $\mathrm{OH}$ radicals generated from ultrafine bubbles collapsed by the application of ultrasound were evaluated from the absorbance measurement using potassium iodide and the behavior observation of sonoluminescence.

\section{Experiment}

\subsection{Preparation of ultrafine bubble water}

An experiment was conducted using ultrapure water (Milli-Q made by Merck Millipore) as water under the environment of class 1000 cleanliness. In addition, for the preparation of ultrafine bubbles, 1 to $100 \mu \mathrm{m}$ microbubbles simultaneously generated as fine bubbles were left to stand for 20 minutes for their floating and separation to eliminate the microbubbles, making only ultrafine bubbles remain. A gas-liquid shear system was used for the generator (Hata, 2012). Moreover, according to the experiment, air, ozone, and argon gas were used for the contained gas, and mixing of impurities other than the gas was prevented by installing a hollow fiber filter $(0.04 \mu \mathrm{m})$ in the introduction path.

\subsection{Measurement of particle size distribution and number density of ultrafine bubbles}

For the measurement of the particle size distribution and number density of ultrafine bubbles, MicrotracBEL Corp. made ZetaView, which is a nanoparticle tracking analysis method, was used.

\subsection{Absorbance measurement using potassium iodide (KI)}

$\mathrm{OH}$ radicals combine with $\mathrm{I}^{-}$dissociated from $\mathrm{KI}$ to become stable in the form of $\mathrm{OH}^{-}$, then the two molecules of $\mathrm{I}^{-}$formed react with $\mathrm{I}_{2}$, and react further $\mathrm{I}^{-}$ to become $\mathrm{I}_{3}{ }^{-}$. That is, ultrafine bubble treatment is performed in the KI solution, and ultrasound is further applied to form $\mathrm{OH}$ radicals, resulting in increasing the concentration of $\mathrm{I}_{3}^{-}$. Since this $\mathrm{I}_{3}^{-}$has an absorption wavelength at $355 \mathrm{~nm}$, the $\mathrm{OH}$ radicals can be confirmed by performing the spectrophotometric measurement for this solution. This time, KI concentration was set to 0.1 mol L-1.

\subsection{Behavior observation of sonoluminescence}

The sonoluminescence observation machine was made by ourselves. A schematic diagram is shown in Figure 1. There is an ultrasonic oscillator $(45 \mathrm{kHz})$ in which a sample (liquid) can be inserted in the lower layer of a dark box, and an optical sensor (this time an image intensifier is used) is installed in the upper layer, composing a system to transmit the signals from the optical sensor to a PC successively, and to visualize and analyze them.

In addition, since the sonoluminescence behavior is affected by the concentration of dissolved gas, first, ultrapure water was deaerated by a vacuum pump, and then only pure oxygen was aerated to make the dissolved oxygen supersaturated. After that, for the behavior observation of sonoluminescence, the contained gas is made to be argon gas (Young, 1976) with which sonoluminescence phenomenon appears strongly, and the gas concentration was made to be constant by using an aeration tube or processing ultrafine bubbles to set the dissolved oxygen amount to $10 \%$ of its saturation value.

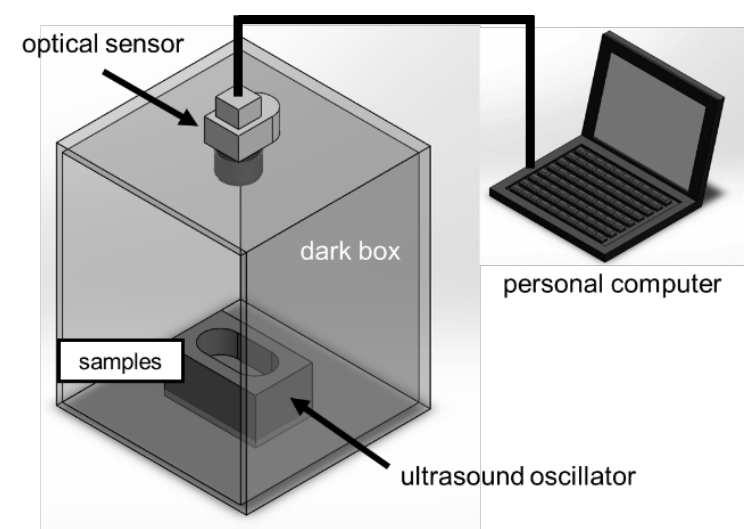

Figure 1. Experimentally made sonoluminescence observation device

\section{Results and Discussion}

\subsection{Measurement of the particle size distribution and number density of ultrafine bubbles}

The particle size distribution of ultrafine bubbles (in ultrafine bubble water) is shown in Figure 2. As a comparison, the particle size distribution of solid particles is shown in Figure 3. This time, particle diameter standard particles (Thermo Fisher Scientific made material: polystyrene latex, average particle diameter: $100 \mathrm{~nm}$ ) with nearly the same size as the diameter of ultrafine bubbles were used as solid impurities. In addition, the number density of standard particles was prepared by dilution so as to approximate the total number density of ultrafine bubbles. As can be seen from Figures 2 and 3 , there is no significant 


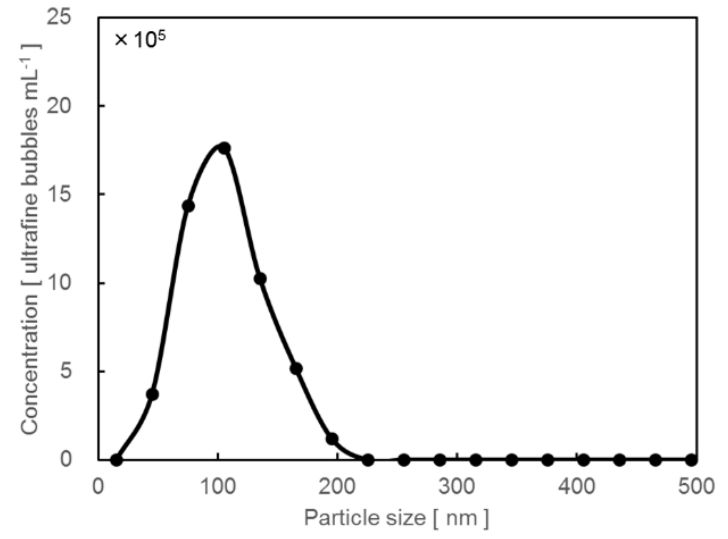

Figure 2. Particle size distribution of ultrafine bubbles.

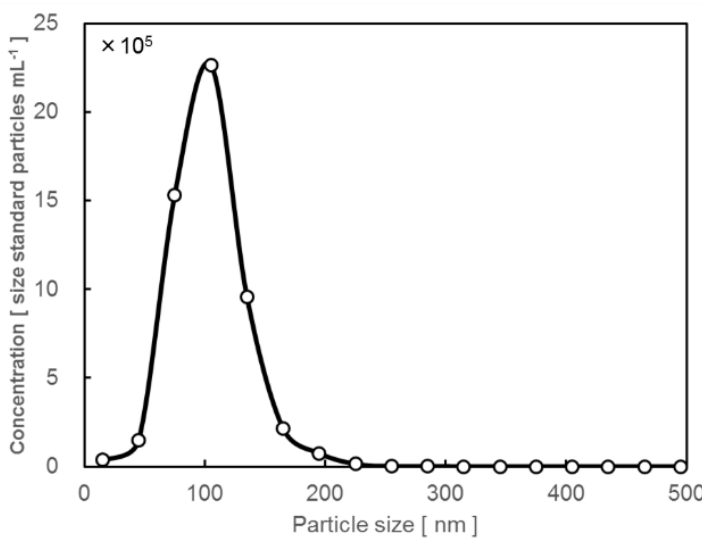

Figure 3. Particle size distribution of particle diameter standard particles (polystyrene latex)

difference except that the standard particles have a higher normal distribution; as seen above, it is difficult to distinguish between ultrafine bubbles and solid particles only by particle size distribution measurement except for the state where the source is clear.

\subsection{Absorbance measurement using potassium iodide (KI)}

When air was used as the introduced gas, the absorption wavelength indicating $\mathrm{I}_{3}^{-}$was observed in the ultrafine bubble water to which ultrasound was applied, confirming the possibility of an evaluation for the presence of ultrafine bubbles using the KI method by making the $\mathrm{OH}$ radicals as an index; on the other hand, its degree of difference was very small, making it difficult to judge the clear difference in the absorbance. Therefore, the amount of $\mathrm{OH}$ radicals generated was increased by using ozone as the contained gas of ultrafine bubbles, trying to enhance the degree of the difference. Ultrasound is applied to ozone water adjusted to the same dissolved ozone concentration by ozoneultrafine bubble water and an aeration tube, and after measuring the respective absorbance spectra, a spectrum obtained by subtracting the spectrum of aeration tube treatment water from the spectrum of ozone-ultrafine bubble water is shown in Figure 4. Also, the ultrasonic frequency was changed to $45 \mathrm{kHz}, 100 \mathrm{kHz}$, and 1.6

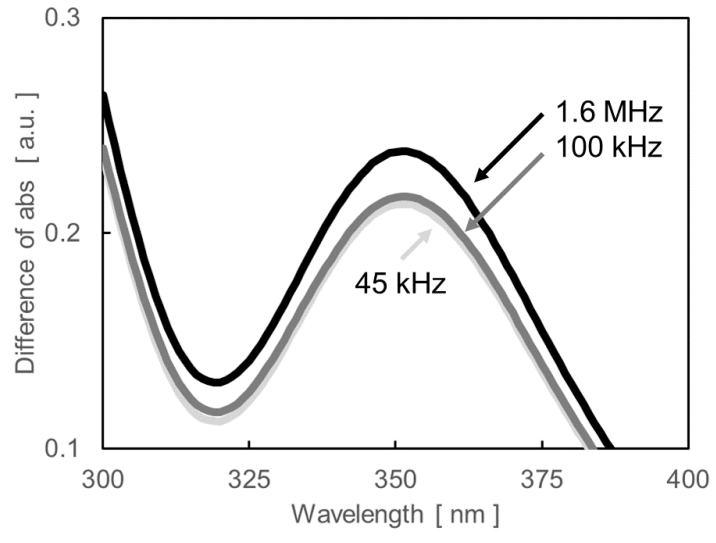

Figure 4. Absorbance spectrum of ozone-ultrafine bubble water to which ultrasound is applied (absorbance spectrum of the system in which ultrasound is applied to ozone water adjusted to the same dissolved ozone concentration by the aeration tube is subtracted)

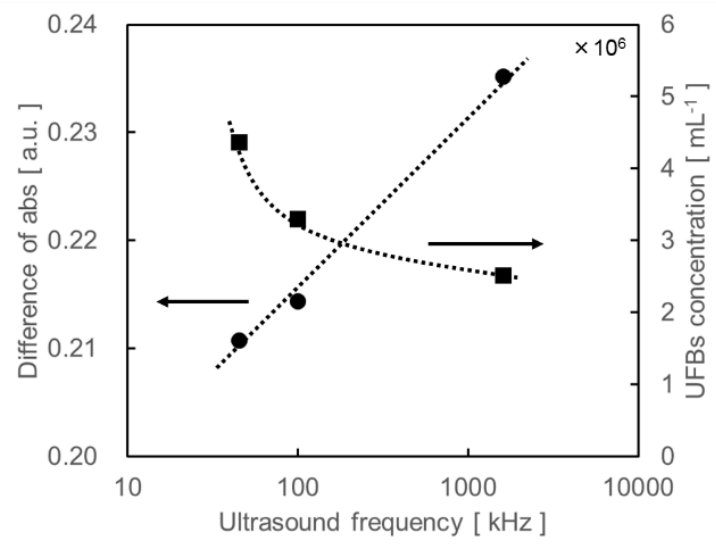

Figure 5. Ultrasound frequency dependence of the number density of ultrafine bubbles and the difference of absorbance spectra at $355 \mathrm{~nm}$ observed after applying ultrasound

MHz. Improvements in the absorbance spectrum at 355 nm corresponding to $\mathrm{I}_{3}{ }^{-}$were observed at all frequencies. Furthermore, Figure 5 shows a diagram plotting the number density of ultrafine bubbles and the difference of absorbance spectra at $355 \mathrm{~nm}$ observed after applying ultrasound against frequency, respectively. The number density of ultrafine bubbles before applying ultrasound was adjusted to $7.2 \times 10^{6} \mathrm{~mL}^{-1}$ at all frequencies. It is found from Figure 5 that the collapse of ultrafine bubbles is accelerated depending on the frequency, and the amount of $\mathrm{OH}$ radicals is increased accordingly.

As a result, stabilization of ultra-fine bubble evaluation by making $\mathrm{OH}$ radicals as an index using the KI method may be expected by using ozone as contained gas of ultra-fine bubbles and using high frequency ultrasound.

\subsection{Behavior observation of sonoluminescence}

Sonoluminescence is a phenomenon in which bubbles are stably expanded and shrunken repeatedly by ultrasound, causing to emit light at each shrink; and in 
multi-bubble sonoluminescence (MBSL), a chemical reaction theory related to hydroxy $(\mathrm{OH})$ radicals has been proposed. In the behavior observation of this sonoluminescence, ultrapure only water, ultrapure water (ultrafine bubble water) to which ultrafine bubble water was added, and ultrapure water to which standard particles were dispersed were used. The sonoluminescence behavior observed immediately after applying ultrasound to each aqueous solution was captured by the observation device of Figure 1 . The image on that occasion is shown in Figure 6 . Sonoluminescence behaviors in ultrapure only water and ultrapure water to which standard particles were added were weak, while strong sonoluminescence behavior was confirmed in ultrafine bubble water.

Furthermore, this sonoluminescence behavior was photographed at a frame number of $30 \mathrm{fps}$ before and after applying ultrasound. Next, by using software that divides the brightness into 256 levels for each frame to count the number of pixels of brightness with a level of 50 or more, the number of pixels of brightness with a level of 50 or more was obtained for the number of frames, and the obtained results are plotted in Figure 7. As shown in this Figure, a strong sonoluminescence behavior occurs at the moment when ultrasound is applied. Even in ultrapure only water and ultrapure water to which standard particles are added, sonoluminescence behavior occurs due to cavitation by ultrasound, but since ultrafine bubble water already contains argonultrafine bubbles, a strong sonoluminescence behavior is

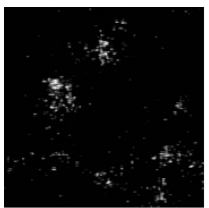

(a)

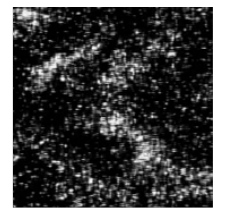

(b)

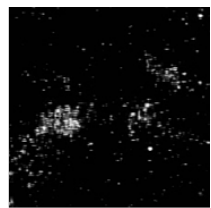

(c)
Figure 6. Observed sonoluminescence behaviors ((a) ultrapure water, (b) ultrafine bubble water, (c) ultrapure water with standard particles dispersed)

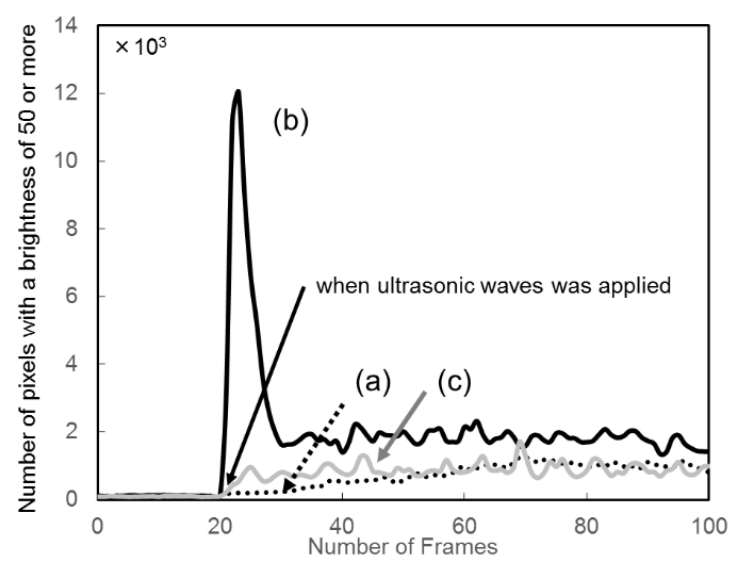

Figure 7. Number of pixels with a brightness of 50 or more is counted from the sonoluminescence observation image and plotted it for each frame ((a) ultrapure water, (b) ultrafine bubble water, (c) ultrapure water with standard particles dispersed)

shown from the collapse immediately after applying ultrasound. As described above, it is difficult to distinguish between ultrafine bubbles and solid particles only by measuring the particle size distribution as shown in Figures 2 and 3; however, combined use of this method has enabled to distinguish between them.

\section{Conclusion}

The method of making $\mathrm{OH}$ radicals generated by the collapse of ultrafine bubbles as an indicator when applying ultrasound was examined as the method of identifying ultrafine bubbles less than $1 \mu \mathrm{m}$ in diameter under the presence of mixed solid nanoparticles.

First, potassium iodide (KI) method was used to evaluate $\mathrm{OH}$ radicals. In the $\mathrm{KI}$ method, when $\mathrm{OH}$ radicals are present, since iodine dissociated from $\mathrm{KI}$ is stabilized as $\mathrm{I}_{3}{ }^{-}$having an absorption wavelength of 355 $\mathrm{nm}, \mathrm{OH}$ radicals generated by measuring this absorbance of $\mathrm{I}_{3}^{-}$with a spectrophotometer can be evaluated. When using air as the contained gas, since the difference in absorbance due to $\mathrm{OH}$ radicals generated by the collapse of ultrafine bubbles is very small, evaluation using the KI method was difficult, but by using ozone as the contained gas, a distinctive difference in absorbance was confirmed by increasing the amount of $\mathrm{OH}$ radicals generated. Furthermore, the higher the ultrasound frequency, the greater the difference in absorbance.

On the other hand, sonoluminescence observation confirmed the luminescence phenomenon related to the collapse of ultrafine bubbles by ultrasound, confirming further that the luminescence intensity of water added with inorganic particles of the same diameter and same number density as ultrafine bubbles was smaller than that of ultrafine bubble water.

In these experiments, since a difference between the experimental results with or without ultrafine bubbles has been confirmed, it will be possible to expect the evaluation or identification of ultrafine bubbles using $\mathrm{OH}$ radicals as an indicator.

\section{Acknowledgements}

This work was supported by JSPS KAKENHI Grant Number $18 \mathrm{~K} 03940$.

\section{References}

Terasaka, K.; "Trends and Challenges of Fine Bubble Technology," Chemical Engineering of Japan, 78, 580584 (2014a)

Ohgaki, K., N. Q. Khanh, Y. Joden, A. Tsuji, and T Nakagawa; "Physicochemical Approach to Nanobubble Solutions," Chemical Engineering Science, 65, 12961300 (2010) 
Uchida, T., S. Oshita, M. Ohmori, T. Tsuno, K. Soejima, S. Shinozaki, Y. Take, and K. Mitsuda; "Transmission Electron Microscopic Observations of Nanobubbles and Their Capture of Impurities in Wastewater," Nanoscale Research Letters, 6, 295-303 (2011)

Kobayashi, H., S. Maeda, M. Kashiwa, and T. Fujita; "Measurements of Ultrafine Bubbles Using Different Types of Particle Size Measuring Instruments," Proceedings Volume 9232, International Conference on Optical Particle Characterization (OPC 2014), doi: 10.1117/12.2064638 (2014)

Leroy, V. and T. Norisuye; "Investigating the Existence of Bulk Nanobubbles with Ultrasound," ChemPhysChem, 17, 2787-279 (2016)

Tada, K., M. Maeda, Y. Nishiuchi, J. Nagahara, T. Hata, Z. Zhuowei, Y. Yoshida, S. Watanabe, and M. Ohmori; "ESR Measurement of Hydroxyl Radicals in Micronanobubble Water," Chemistry Letters, 43, 1907-1908 (2014)

Yasui, K., T. Tuziuti, and W. Kanematsu; "Extreme Conditions in a Dissolving Air Nanobubble," Physical Review E, 94, 013106, 13 pages (2016)

Hata, T.; "Super-micro Bubble Generator," WO Patent, 2012/105536 (2012)

Young, F. R.; "Sonoluminescence from Water Containing Dissolved Gases," The Journal of the Acoustical Society of America, 60, 100-104 (1976) 